\section{AUTHOR INDEX TO ABSTRACTS}

(Numbers are abstract numbers)

Adebonojo, F. O., 50

Anttila, R. O., 33

Aronson, A. S., 9

Bachmann, C., 54, 55

Ballowitz, L., 78

Balsan, S., 17

Baud, R., 15

Baumgartner, R., 54, 55

Barltrop, D., 88

Barrat, T. M., 33

Bartholome, K., 52

Bendien, C., 68

Beno, P., 83, 95

Berend, K., 91

Bhakdi, S., 29

Bieler, E., 99

Bienzle, U., 29

Blum, A., 66

Boda, D., 27

Bommer, J., 80

Boyd, R., 26

Brandis, C. M., 3

Brauser, B., 58

Bremer, H. J., 45

Bretscher, D., 24

Brodehl, J., 2, 3

Broyer, M., 32, 39, 82

Brun Del Re, S., 66

Bryan, E. M., 89

Byrd, D. J., 53

Callis, L., 8, 81

Cantz, M., 43, 92

Carson, J., 49, 59

Castello, F., 8, 81

Chappuis, M. C., 20

Chiswick, M. L., 61

Cser, A., 74

Curran, P. F., 22, 64

Cvoric, A., 10

Danesh, B. J. N. Z., 4

Dartois, A. M., 17

David, L., 20

Day, N. K., 34

Dechaux, M., 39

De Jong, M. C. J., 12

Delaporte, C., 82

Delvin, E. E., 1

Demant, F., 83

Der Kaloustian, V. M., 65
Desjeux, J. F., 22, 64

Deyhle, P., 66

Dillon, M. J., 40

Djatchcova, A., 84

Dober, I., 74

Dobos, M., 90

Doehmann, U., 48

Donath, A., 15

Dubowitz, V., 56

Eck, E., 27

Eeg Olofsson, 0., 72

Egli, F., 42

Eicher, E. M., 23

Elema, J. D., 68

Elmiger, P., 42

Endreffy, E., 27

Enger, E., 25

Fallstrom, K., 72

Federlin, K., 18

Fekete, G., 90

Fosbrooke, A., 102

Francois, R., 20

Franz, H. E., 18

Gacs, G., 91

Garcia, Fuentes, M., 7

Gautier, E., 6

Gehler, J., 43, 92

Geiger, H., 34

Genton, N., 11

Gerber, A., 49

Gimpert, E., 48

Gitzelmann, R., 69

Gjessing, L. R., 94

Glorieux, F. H., 1, 23

Godbole, V., 97

Goldis, G., 35

Good, R. A., 34

Gordon, H. A., 86

Guignard, J. P., 6, 11

Grieder, H. R., 66

Hadorn, B., 24, 49

Hagmann, R., 24

Hamberg, M., 60

Haljamäe, H., 25

Hanefeld, F., 78

Harvey, D. R., 97
Emilio, M. G., 28

Gane, R., 35
Hatemi, N., 39

Haworth, C. A., 26

Helmke, K., 18

Heim, T., 74

Heinonen, K., 79

Hers, H. G., 70

Herschkowitz, N., 44, 59

Hirvonen, L., 63

Hitzig, W. H., 48

Hokegard, K. H., 76

Holmberg, C., 38

Hommes, F. A., 68

Houston, I. B., 4

Howald, B., 24

Hrbek, A., 76

Hue, L., 70

Ignatova, M. S., 84

Indermuhle, P., 15

Jacobsen, J., 77

Jakel, A., 3

Jaszai, V., 74

Jeschke, R., 18

Jouja, V., 67

Kaiser, D., 47

Kalafatic, Z., 87

Karlsson, K., 76

Kjellmer, I., 76

Kleihauer, E., 29

Knufermann, H., 29

Kochen, W., 53

Kohlschuetter, A., 59

Krempien, B., 14

Krohn, H. P., 2

Lacourt, G., 99

Lenard, H. G., 57

Liappis, N., 3

Linblad, B. S., 93

Lind, J., 75

Lindemann, R., 94

Link, E., 18

Lloyd, J. K., 73

Lodinova, R., 67

Loirat, C., 32

Lombeck, I., 45

Lund, H. T., 77

Luthi, C., 24

Lutz, P., 52 
Macken, J., 36

Manz, F., 5

Mathéová, E., 83, 95

Mayor, G., 11

McIntosh, N., 13

Mehes, K., 19

Mehls, O., 5, 14, 80

Menano, H. P., 28

Milner, R. D. G., 61, 96

Monn, E., 41

Monnens, L. A. H., 12

Morin, C. C., 1

Muranyi, L., 27

Murib, A., 65

Mushin, A. S., 62

Nergardh, A., 75

Niethammer, D., 29, 31

Nussle, D., 15

Oates, R. K., 97

O'Brien, J. F., 43

Oetliker, O., 24

Osnes, J. B., 41

Oye, I., 41

Pantelakis, S. N., 98

Pasternak, A., 38

Paunier, L., 21, 99

Pavkovcekova, O., 83, 95

Peltonen, R., 63

Peltonen, T., 63

Perheentupa, J., 38, 46

Petersen, I., 77

Piazolo, P., 18

Pluess, H. J., 48

Poirier, R., 1

Popovic-Rolovic, M., 85

Proesmans, W., 36

Przyrembel, H., 45
Rall, T. W., 71

Räihä, N. C. R., 71, 79

Rennert, O. M., 47

Richards, B., 96

Ricour, C., 17, 39

Ritz, E., 14, 80

Rodriguez Soriano, J., 7

Rosen, K. G., 76

Rudiger, H. W., 51

Rumpelt, H. J., 37

Ryness, J., 40

Samuelsson, B., 60

Sbirakis, S., 98

Schaffner, T., 66

Schneider, A., 24

Schneider, P., 21

Schroter, W., 30

Schuler, D., 90

Schuster, E., 87

Schwabe, U., 2

Schwartz, A. L., 71

Scriver, C. R., 23

Seip, M., 94

Seriogina, V., 84

Shaw, J. C. L., 13

Sigstrom, L., 25

Simell, O., 46

Sinaniotis, C. A., 98

Sizonenko, P. C., 21, 99

Smith, A., 88

Soothill, J. F., 33

Southard, J. L., 23

Sovik, O., 101

Spranger, J., 43, 92

Stacy, T. E., 26

Stalder, G., 42

Steendijk, R., 16

Steinmann, B., 69

Stern, C. M. M., 100
Strandberg, K., 60

Ströder, J., 18

Svenningsen, N. N., 9

Szelid, Z. S., 19

Taghizadeh, A., 13

Tai, Y. H., 22, 64

Thoenes, W., 37

Tischler, V., 83, 95

Tolksdorf, C. M., 43, 92

Torrado, A., 6, 11

Travers, R., 1, 23

Tuvemo, T., 60

Ungefehr, K., 30

Vallo, A., 7

Van Damme, B., 36

Van Den Berghe, G., 70

Van Wieringen, P. M. V.,

Varga, F., 74

Versmold, H., 58

Vidnes, J., 101

Vila, A., 8, 81

Vischer, D., 48

Visy, M., 86

Vlatkovic, G., 87

Wakid, N. W., 65

Ward, R. H. T., 26

Wendel, U., 45, 51

Wentrup, H., 51

Westcott, S., 61

West, R. J., 73

Wharton, B. A., 102

Wiesmann, U. N., 44, 59

Wyss, M., 21

Zahnd, G., 99

Zurbrugg, R. P., 66 